\title{
О возможности распространения уединенной электромагнитной волны в биграфене
}

\author{
(C) Е.И. Кухарь ${ }^{1,2}$, С.В. Крючков ${ }^{1,2}$, Е.С. Ионкина ${ }^{2}$ \\ ${ }^{1}$ Волгоградский государственный социально-педагогический университет, \\ 400066 Волгоград, Россия \\ ${ }^{2}$ Волгоградский государственный технический университет, \\ 400005 Волгоград, Россия \\ E-mail: eikuhar@yandex.ru
}

(Получена 10 мая 2017 г. Принята к печати 22 июня 2017 г.)

Изучена возможность распространения уединенной электромагнитной волны в биграфене, между слоями которого приложена разность потенциалов. Найдено условие, при котором возможно формирование такой волны. Исследована зависимость формы уединенной волны от разности потенциалов между графеновыми слоями. В качестве возможной идентификации уединенной волны вычислен электрический заряд, увлекаемый волной в направлении своего распространения.

DOI: 10.21883/FTP.2018.06.45926.8634

\section{1. Введение}

В последнее время в области физики твердого тела учеными большое внимание уделяется не только однослойному, но и двуслойному графену (биграфену) [1-4]. Развитие этой области наноэлектроники стало возможным благодаря возможностям создания и обработки таких материалов при помощи методов современной литографии $[5,6]$. Интерес к графеновым структурам связан с их удивительными транспортными свойствами. В частности, для графена характерны высокая подвижность носителей заряда и высокое значение коэффициента теплопроводности. Непараболичность закона дисперсии, обнаруженная в графене и биграфене, приводит к нелинейному терагерцовому и оптическому откликам [7-9]. В частности, в $[10,11]$ предлагалось использовать графен и биграфен для генерации третьей гармоники.

Изучение электродинамических свойств графеновых структур имеет как прикладное $[12,13]$, так и фундаментальное значение $[2,3,14]$. Закон дисперсии носителей заряда в однослойном графене вблизи так называемых дираковских точек линеен по абсолютной величине квазиимпульса, что позволяет использовать графен для проверки релятивистских эффектов [1-3]. Изучение взаимодействий дираковских электронов с элементарными возбуждениями привело к предсказанию и наблюдению новых особенностей коллективных явлений в графеновых структурах $[15,16]$.

Графеновые структуры могут также использоваться в качестве рабочей среды для генерации ультракоротких электомагнитных (ЭМ) импульсов [12], имеющих ряд приложений [17,18]. В работе [19] изучена возможность генерации уединенных ЭМ волн нового типа в сверхрешетках на основе графена (ГСР). Однако для этого необходимо изготавливать ГСР, в зонной структуре которых имелись бы узкие разрешенные зоны (минизоны), разделенные достаточно широкими запрещенными зонами. Исследование возможностей изготовления таких структур, а также изучение их зонных характеристик имеют пока теоретический характер [20]. Тем не менее, при определенных условиях в качестве активной среды для генерации уединенной волны можно использовать биграфен, между слоями которого приложена разность потенциалов. При этом, как показано далее, конечности зоны проводимости не требуется.

Одним из свойств зонной структуры биграфена является возможность открытия энергетической щели за счет приложения постоянного электрического поля в направлении, перпендикулярном графеновым слоям [7,21-24]. В этом случае энергетическое расстояние между валентной зоной и зоной проводимости контролируется путем изменения разности потенциалов между слоями [21,24], что является важным с точки зрения приложений в нано- и оптоэлектронике. Кроме того, в такой ситуации минимум энергии смещается из дираковской точки зоны Бриллюэна. В центре же этой зоны возникает область отрицательных эффективных масс $[4,7,21,22,25]$. Далее показано, что эта особенность зонной структуры биграфена приводит к возможности генерации уединенного ЭМ $0 \pi$-импульса.

\section{2. Параметры зонной структуры биграфена}

Наличие разности потенциалов между слоями биграфена приводит к наведению энергетической щели $\Delta_{g}$ между валентной зоной и зоной проводимости. Изменение разности потенциалов позволяет контролировать величину $\Delta_{g}$, что дает возможность управления межзонными переходами и концентрацией носителей заряда $[21,26]$. В частности, возможно подавление межзонных переходов за счет соответствующего подбора значения внешнего напряжения $[11,27]$. С точки зрения возможности создания устройств с перестраиваемыми 


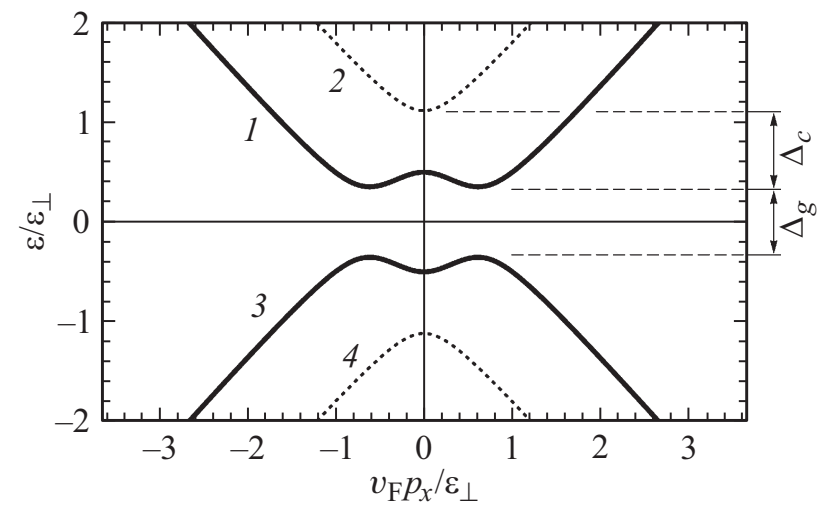

Рис. 1. Дисперсионные линии, построенные по формуле (1) при $V_{0}=\varepsilon_{\perp}$.

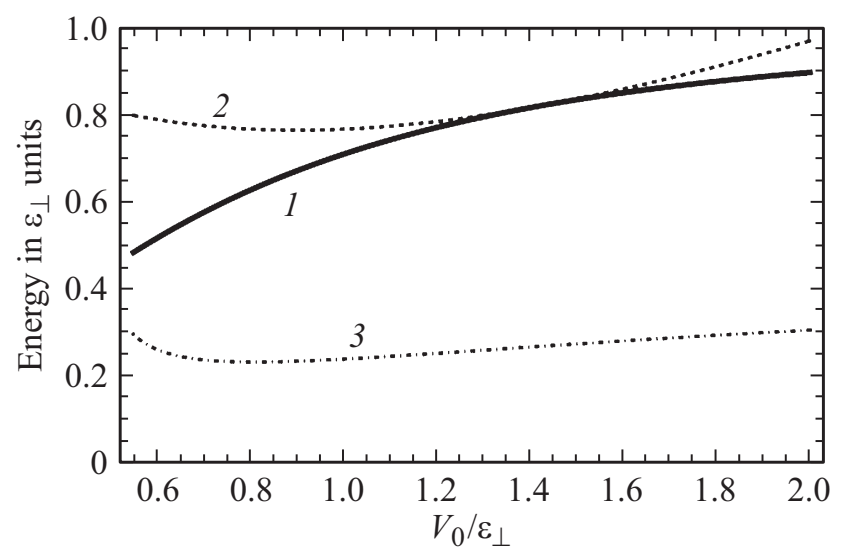

Рис. 2. Зависимость энергетической щели $\Delta_{g}$ от $V_{0}(1)$; зависимость параметра $\Delta_{c}$ от $V_{0}(2)$; зависимость энергии, приобретаемой электроном в поле уединенной волны, от $V_{0}(3)$.

характеристиками описанная особенность обеспечивает преимущество биграфена перед однослойным графеном.

Приведем здесь функциональную зависимость параметров зонной структуры от величины разности потенциалов между графеновыми слоями. Электронный спектр биграфена в поперечном постоянном электрическом поле имеет вид [21]

$$
\varepsilon(\mathbf{p})= \pm \sqrt{\frac{\varepsilon_{\perp}^{2}}{2}+\frac{V_{0}^{2}}{4}+v_{\mathrm{F}}^{2} \mathbf{p}^{2} \mp \sqrt{\frac{\varepsilon_{\perp}^{4}}{4}+\left(\varepsilon_{\perp}^{2}+V_{0}^{2}\right) v_{\mathrm{F}}^{2} \mathbf{p}^{2}}}
$$

где $V_{0}$ - параметр, определяющийся внешним напряжением, 0.39 эВ. Формула (1) описывает четыре дисперсионные ветви: две ветви в зоне проводимости (рис. 1, линии 1 и 2) и две ветви в валентной зоне (рис. 1, линии 3 и 4). Считаем, что валентная зона полностью заполнена так, что вклад в проводимость электроны из этой зоны не дают. Кроме того, температура системы предполагается настолько низкой, что носители заряда сосредоточены на нижней дисперсионной ветви 1 (рис. 1). Энергетическая щель $\Delta_{g}$, возникающая между валентной зоной и зоной проводимости в поперечном поле, равна

$$
\Delta_{g}=\frac{\varepsilon_{\perp} V_{0}}{\sqrt{\varepsilon_{\perp}^{2}+V_{0}^{2}}}
$$

Энергетическое расстояние между минимумами линий 1 и 2 (рис. 1) дается формулой

$$
\Delta_{c}=\frac{\varepsilon_{\perp}}{2}\left(\sqrt{4+\frac{V_{0}^{2}}{\varepsilon_{\perp}^{2}}}-\frac{V_{0}}{\sqrt{\varepsilon_{\perp}^{2}+V_{0}^{2}}}\right) .
$$

Зависимости параметров $\Delta_{g}$ и $\Delta_{c}$ от $V_{0}$ показаны на рис. 2 (линии 1 и 2 соответственно).

\section{3. Генерация уединенной ЭМ волны}

Пусть в биграфене создано дополнительное ЭМ поле так, что вектор напряженности электрической составляющей направлен вдоль графеновых слоев. Считаем, что энергия, передаваемая электрону этим полем, удовлетворяет неравенствам $\varepsilon_{e l}<\Delta_{g}, \Delta_{c}$. Эти условия могут быть достигнуты соответствующим изменением параметра $V_{0}$. В этом случае в законе дисперсии (1) достаточно оставить только верхние знаки, записав

$$
\varepsilon(\mathbf{p})=\varepsilon_{\perp} \chi\left(\frac{v_{\mathrm{F}} p_{x}}{\varepsilon_{\perp}}, \frac{v_{\mathrm{F}} p_{y}}{\varepsilon_{\perp}}, \frac{V_{0}}{\varepsilon_{\perp}}\right),
$$

где введена следующая функция:

$$
\chi\left(q_{x}, q_{y}, u\right)=\sqrt{\frac{1}{2}+\frac{u^{2}}{4}+q_{x}^{2}+q_{y}^{2}-\sqrt{\frac{1}{4}+\left(1+u^{2}\right)\left(q_{x}^{2}+q_{y}^{2}\right)} .}
$$

Существенная непараболичность закона дисперсии (4) приводит к тому, что биграфен может быть использован в качестве оптически нелинейной рабочей среды [7-9]. В частности уравнение самосогласованного ЭМ поля, учитывающее электрический ток, наводимый этим же полем в данной среде, оказывается нелинейным.

Вычислим плотность тока $j_{x}$, возникающую в биграфене под действием продольного электрического поля. В бесстолкновительном режиме имеем

$$
j_{x}=-e \sum_{\mathbf{p}} f_{0}(\mathbf{p}) v_{x}\left(\mathbf{p}+\frac{e}{c} \mathbf{A}\right),
$$

где $f_{0}(\mathbf{p})$ - равновесная функция распределения, $v_{x}=\partial \varepsilon / \partial p_{x}, \mathbf{A}=(A, 0)-$ векторный потенциал поля. Для вычисления плотности тока (6) разложим выражение, стоящее под знаком суммы, в ряд по параметpy $v_{\mathrm{F}} \varphi / c$ :

$$
j_{x}=-e v_{\mathrm{F}} \sum_{\mathbf{p}} f_{0}(\mathbf{p}) \sum_{n} \frac{1}{n !}\left(\frac{v_{\mathrm{F}} \varphi}{c} \frac{\partial}{\partial q_{x}}\right)^{n} \frac{\partial \chi}{\partial q_{x}},
$$

где $\varphi=e A / \varepsilon_{\perp}-$ безразмерный потенциал поля, $\mathbf{q}=v_{\mathrm{F}} \mathbf{p} / \varepsilon_{\perp}$. Считая, что $v_{\mathrm{F}} \varphi / c \ll 1$, отбросим в сумме (7) слагаемые, степени которых превышают $n=3$, и учтем следующее соотношение: 
$\partial_{q_{x}}^{n} \chi\left(q_{x} q_{y}\right)=(-1)^{n} \partial_{q_{x}}^{n} \chi\left(-q_{x},-q_{y}\right)$. В результате суммирования по квазиимпульсу в (7) получим

$$
j_{x}=-\frac{e n_{0} v_{\mathrm{F}}^{2} \alpha_{1}}{c}\left(\varphi+\frac{v_{\mathrm{F}}^{2}}{6 c^{2}} \frac{\alpha_{2}}{\alpha_{1}} \varphi^{3}\right)
$$

Здесь $n_{0}-$ концентрация свободных носителей заряда в биграфене,

$$
\begin{gathered}
\alpha_{1}(u)=\frac{1}{\beta(u)} \sum_{\mathbf{q}} \frac{\partial^{2} \chi}{\partial q_{x}^{2}} e^{-\frac{\varepsilon \perp \chi\left(q_{x}, q_{y}, u\right)}{k T}}, \\
\alpha_{2}(u)=\frac{1}{\beta(u)} \sum_{\mathbf{q}} \frac{\partial^{4} \chi}{\partial q_{x}^{4}} e^{-\frac{\varepsilon \perp \chi\left(q_{x}, q_{y}, u\right)}{k T}}, \\
\beta(u)=\sum_{\mathbf{q}} e^{-\frac{\varepsilon_{\perp} \chi\left(q_{x}, q_{y}, u\right)}{k T}},
\end{gathered}
$$

$T$ - температура. В пределе низких температур $k T \ll \varepsilon_{\perp}$ имеем

$$
\begin{gathered}
\alpha_{1}(u)=\frac{u\left(2+u^{2}\right)}{\left(1+u^{2}\right)^{3 / 2}}, \\
a_{2}(u)=\frac{6\left(4-u^{2}\left(2+u^{2}\right)\left(6+11 u^{2}+7 u^{4}+u^{6}\right)\right)}{u\left(1+u^{2}\right)^{9 / 2}} .
\end{gathered}
$$

Величина $V_{0}$ должна быть такой, чтобы значения функции $\alpha_{2}$ были достаточно далеки от нуля. Графики функций $\alpha_{1}(u)$ и $\alpha_{2}(u)$ показаны соответственно на рис. 3 и 4 для различных температур. Как видно из рис. 4 , функция $\alpha_{2}(u)$ меняет знак на отрицательный при определенном значении параметра $V_{0}$. При $T=4.2 \mathrm{~K}$ смене знака соответствует $V_{0}=V_{g} \sim 0.2$ эВ.

Далее рассмотрим случай, когда $\alpha_{2}<0$. Из рис. 3 и 4 находим, что в области значений величины $V_{0}>V_{g}$, для которых $\alpha_{2}<0$, параметры $\alpha_{1}$ и $\left|\alpha_{2}\right|$ имеют порядок $\alpha_{1} \sim 1,\left|\alpha_{2}\right| \sim 10$, и вклад кубического по потенциалу $\varphi$ слагаемого в (8) существен, если напряженность электрического поля, описываемого этим потенциалом, достигает порядка $10^{4} \mathrm{~B} / \mathrm{cm}$. При этом вклад слагаемых суммы (7) с $n>3$ мал, и пренебрежение последними оправдано.

После подстановки (8) в уравнение для потенциала ЭМ поля $\left(\partial_{t}^{2}-c^{2} \partial_{y}^{2}\right) A_{x}=4 \pi c j_{x}$ получаем

$$
\frac{\partial^{2} \varphi}{\partial t^{2}}-c^{2} \frac{\partial^{2} \varphi}{\partial y^{2}}+\omega_{p l}^{2}\left(\varphi-\mu \varphi^{3}\right)=0
$$

где $\omega_{p l}=2 e v_{\mathrm{F}} \sqrt{\pi n_{0} / \varepsilon_{\perp} d}-$ плазменная частота, $d-$ расстояние между графеновыми слоями, $\mu=$ $=-v_{\mathrm{F}}^{2} \alpha_{2} / 6 c^{2}>0$, а также учтено, что в режиме генерации $\alpha_{1} \sim 1$.

Введем следующие переменные: $\xi=\left(y-v_{p} t\right) / L_{p}$, $L_{p}^{2}=\left(c^{2}-v_{p}^{2}\right) / \omega_{p l}^{2}$. Тогда из (12) получаем

$$
\frac{d^{2} \varphi}{d \xi^{2}}-\varphi+\mu \varphi^{3}=0
$$

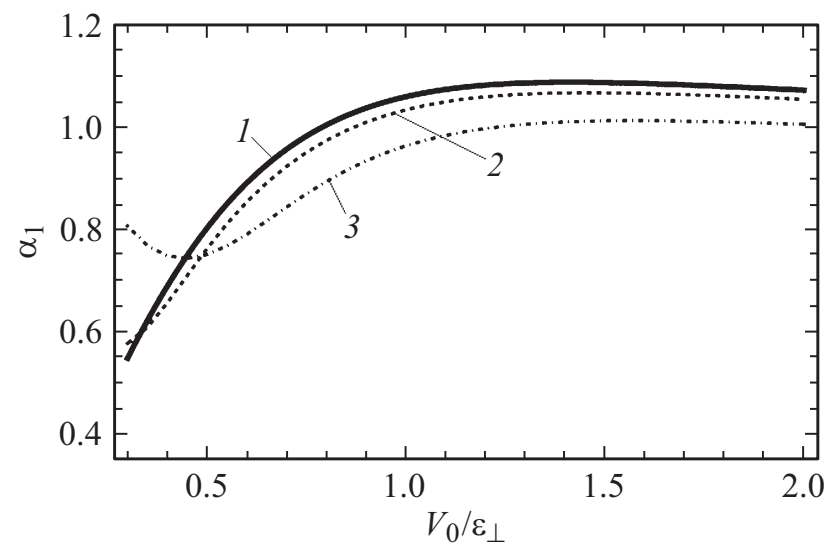

Рис. 3. Зависимость коэффициента $\alpha_{1}$ от $V_{0}$ при температурах $T=0$ K (1), 20 (2) и $77.4 \mathrm{~K}(3)$.

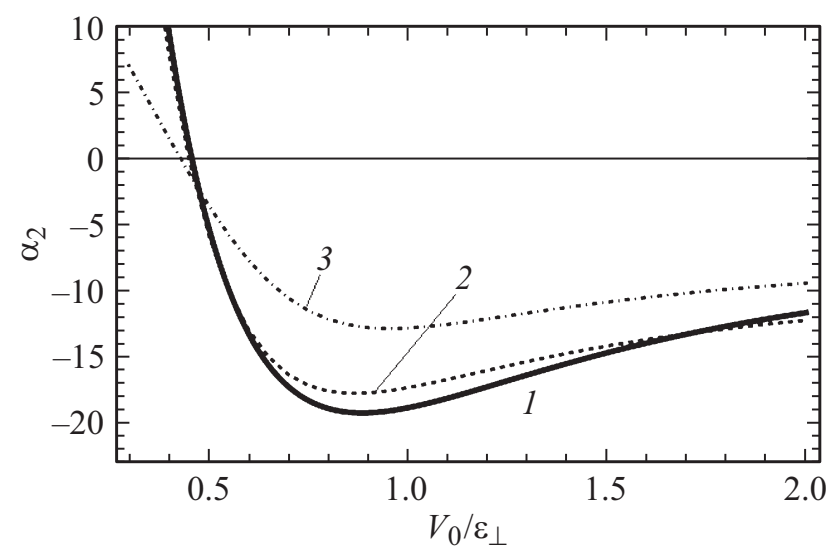

Рис. 4. Зависимость коэффициента $\alpha_{2}$ от $V_{0}$ при температурах $T=0 \mathrm{~K}(1), 4.2(2)$ и $77.4 \mathrm{~K}(3)$.

Уравнение (13) совпадает по форме с уравнением движения частицы в двойной потенциальной яме (маятник Дуффинга) [28], где роль времени играет безразмерная переменная $\xi$. Сепаратрисное решение этого уравнения хорошо известно [28] и имеет вид $\varphi_{s}=\sqrt{2 / \mu} \mathrm{ch}^{-1} \xi$. В рассматриваемой ситуации данное решение описывает распространение уединенного ЭМ импульса, напряженность электрического поля которого равна

$$
E_{x}(y, t)=-\frac{E_{0}}{\sqrt{\left|\alpha_{2}\right|}} \operatorname{sh}\left(\frac{y-v_{p} t}{L_{p}}\right) \operatorname{ch}^{-2}\left(\frac{y-v_{p} t}{L_{p}}\right),
$$

где $E_{0}=2 \sqrt{3} \varepsilon_{\perp} v_{p} / e v_{\mathrm{F}} L_{p}, \quad v_{p}-$ скорость импульса. Таким образом, если выполняется неравенство $\alpha_{2}\left(V_{0} / \varepsilon_{\perp}\right)<0$, то в биграфене возможна генерация уединенной ЭМ волны. Форма этой волны является антисимметричной относительно точки $\xi=0$ (рис. 5), что характерно для $0 \pi$-импульсов. Из рис. 5 видно, что амплитуда волны растет с увеличением параметра $V_{0}$.

Для скорости ЭМ импульса $v_{p} \sim 10^{10} \mathrm{~cm} / \mathrm{c}$ и следующих типичных параметров структуры $[25,29]$ : $V_{0}=\varepsilon_{\perp}=0.39$ эВ $, d=0.34 \mathrm{Hм}, n_{0} \sim 10^{9} \mathrm{~cm}^{-2}$, плазменная частота равна $\omega_{p l}=3 \cdot 10^{13} \mathrm{c}^{-1}$, а амплитуда вол- 


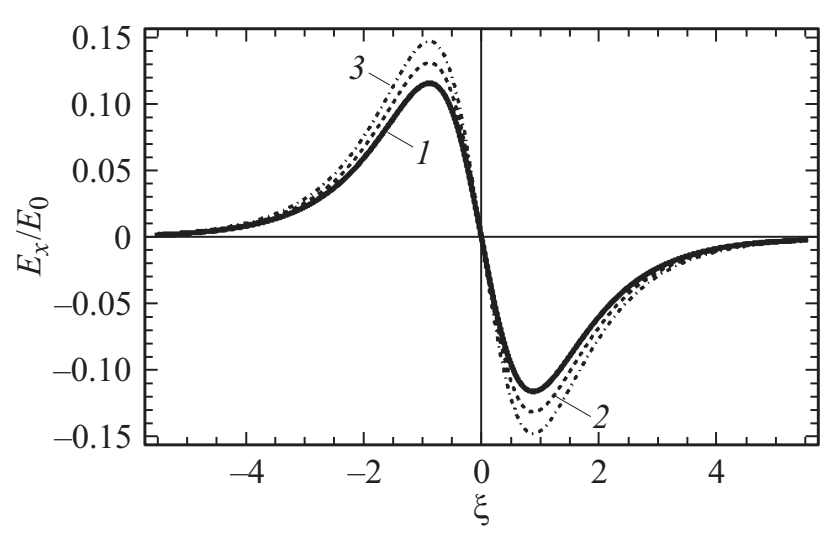

Рис. 5. Профиль уединенной ЭМ волны, распространяющейся в биграфене: $V_{0}=0.39(1), 0.58(2), 0.78$ эВ (3).

ны составляет порядок $10^{4} \mathrm{~B} / \mathrm{cM}$. Энергия, приобретаемая электроном в поле ЭМ волны, имеет порядок $\varepsilon_{e l} \sim e E_{\max } v_{\mathrm{F}} \tau_{p}$, где $E_{\max }-$ амплитуда ЭМ импульса, зависящая от параметра $V_{0}, \tau_{p}=L_{p} / v_{p}$ - время прохождения ЭМ импульса. Зависимость $\varepsilon_{e l}$ от $V_{0}$ показана на рис. 2 (линия 3 ), из которого видно, что энергия электронов не превосходит значения параметров (2) и (3). Поэтому использованное выше приближение, в котором учитывается только нижняя дисперсионная линия зоны проводимости (линия 1 на рис. 1), оправдано.

\section{4. Эффект увлечения, вызванный уединенной волной в биграфене}

Одним из проявлений существования уединенной ЭМ волны может выступить эффект увлечения электронов такой волной [19] - возникновение электрического тока в направлении распространения волны. Природа этого эффекта сходна с природой радиоэлектрического эффекта и объясняется как результат передачи импульса ЭМ волной электронной подсистеме. В отсутствие столкновений с неоднородностями решетки плотность тока увлечения, обусловленного передачей импульса электронной подсистеме ГСР, может быть найдена с помощью формулы

$$
j_{y}=-e \sum_{\mathbf{p}} f_{0}(\mathbf{p}) v_{y}\left(p_{x}, p_{y}+p_{1}\right),
$$

где $v_{y}=\partial \varepsilon / \partial p_{y}, p_{1}-$ средний импульс электрона, равный, согласно закону сохранения импульса, плотности переданного электронной подсистеме импульса ЭМ поля волны, отнесенного к концентрации электронов: $p_{1}=E_{x}^{2} d / 4 \pi v_{p} n_{0}$.

Оценка, выполненная для указанных выше параметров структуры и уединенной волны, показывает, что $p_{1} v_{\mathrm{F}} / \varepsilon_{\perp} \ll 1$. С учетом последнего неравенства выражение для плотности тока увлечения примет вид

$$
j_{y}=-\frac{e v_{\mathrm{F}}^{2} E_{x}^{2}}{4 \pi v_{p} \varepsilon_{\perp}} .
$$

С помощью (15) вычисляем заряд, увлекаемый уединенной волной (14) в направлении своего распространения через единицу площади поперечного сечения:

$$
Q=-\frac{Q_{0}}{\left|\alpha_{2}\left(V_{0} / \varepsilon_{\perp}\right)\right|},
$$

где $Q_{0}=2 \varepsilon_{\perp} / \pi e L_{p} \sim 3.6$ пКл/мм². Последний результат показывает, что величиной переносимого волной заряда можно управлять путем изменения разности потенциалов между слоями биграфена.

\section{5. Заключение}

Выше показано, что в биграфене возможно распространение уединенной ЭМ волны, если параметр $V_{0}$, определяемый разностью потенциалов между графеновыми слоями, превышает значение $V_{g}$, соответствующее смене знака параметра $\alpha_{2}$, определяемого второй формулой (9). При низких температурах $(\sim 4.2 \mathrm{~K})$ это значение составляет $V_{g} \sim 0.2$ эВ. Расчет, выполненный в бесстолкновительном приближении, показал, что в этом случае уравнение д’Аламбера, записанное для ЭМ поля волны с учетом электрического тока, наводимого данным полем, приобретает вид (12). Решение последнего сводится к решению уравнения маятника Дуффинга (13), которое и определяет форму уединенной волны, характерную для $0 \pi$-импульсов (рис. 5). При $V_{0}=0.39$ эВ и $T=4.2 \mathrm{~K}$ амплитуда напряженности электрического поля волны составляет $10^{4} \mathrm{~B} / \mathrm{cm}$, а увлекаемый волной в направлении своего распространения электрический заряд, отнесенный к площади поперечного сечения, составляет 3.6 пКл/мм ${ }^{2}$, что вполне измеримо экспериментально.

Преимуществом биграфена перед ГСР являются следующие обстоятельства. Во-первых, изготовление биграфена является менее трудоемким процессом по сравнению с созданием дополнительного периодического потенциала на графеновом листе. Во-вторых, не любой дополнительный периодический потенциал, формируемый в графеновом монослое, приводит к образованию в энергетическом спектре мини-зон проводимости, разделенных запрещенными зонами. Наличие же узких мини-зон является необходимым условием генерации уединенных ЭМ волн в структурах со сверхрешетками. В то же время, как показано выше, для генерации такой волны в биграфене не требуется конечности зоны проводимости. Уединенная волна может формироваться за счет области отрицательных эффективных масс в центре зоны Бриллюэна, возникающей в случае, если между графеновыми слоями приложена конечная разность потенциалов.

Работа выполнена при финансовой поддержке Министерства образования и науки России на выполнение государственных работ в сфере научной деятельности в рамках проектной части государственного задания, код проекта: 3.2797.2017/4.6. 


\section{Список литературы}

[1] Y. Zhang, Y.-W. Tan, H.L. Stormer, P. Kim. Nature, 438, 201 (2005).

[2] M.I. Katsnelson, K.S. Novoselov, A.K. Geim. Nat. Phys., 2, 620, (2006)

[3] A. Ghazaryan, T. Chakraborty, P. Pietiläinen. J. Phys.: Condens. Matter, 27, 185301 (2015).

[4] E. McCann, V.I. Fal'ko. Phys. Rev. Lett., 96, 086805 (2006).

[5] W.-K. Lee, J.T. Robinson, D. Gunlycke, R.R. Stine, C.R. Tamanaha, W.P. King, P.E. Sheehan. Nano Lett., 11, 5461 (2011).

[6] F. Bonaccorso, A. Lombardo, T. Hasan, Z. Sun, L. Colombo, A.C. Ferrari. Mater. Today, 15, 564 (2012).

[7] E.J. Nicol, J.P. Carbotte. Phys. Rev. B, 77, 155409 (2008).

[8] R.R. Hartmann, J. Kono, M.E. Portnoi. Nanotechnology, 25, 322001 (2014).

[9] S. Sekwao, J.P. Leburton. Appl. Phys. Lett., 106, 063109 (2015).

[10] I. Al-Naib, J.E. Sipe, M.M. Dignam. Phys. Rev. B, 90, 245423 (2014).

[11] R. McGouran, I. Al-Naib, M.M. Dignam. Phys. Rev. B, 94, 235402 (2016).

[12] D. Popa, Z. Sun, F. Torrisi, T. Hasan, F. Wang, A.C. Ferrari. Appl. Phys. Lett., 97, 203106 (2010).

[13] Д.А. Свинцов, В.В. Вьюрков, В.Ф. Лукичёв, А.А. Орликовский, А. Буренков, Р. Охснер. ФТП, 47, 244 (2013).

[14] V.M. Apalkov, T. Chakraborty. Phys. Rev. Lett., 105, 036801 (2010).

[15] Y.E. Lozovik, A.A. Sokolik. Phys. Lett. A, 374, 2785 (2010).

[16] A. Bostwick, F. Speck, T. Seyller, K. Horn, M. Polini, R. Asgari, A.H. MacDonald, E. Rotenberg. Science, 328, 999 (2010).

[17] С.В. Крючков, Е.В. Капля. ЖТФ, 48, 53 (2003).

[18] Z. Sun, T. Hasan, A.C. Ferrari. Physica E, 44, 1082 (2012).

[19] S.V. Kryuchkov, E.I. Kukhar'. Physica B, 408, 188 (2013).

[20] П.В. Ратников. Письма ЖЭТФ, 90, 515 (2009).

[21] E. McCann. Phys. Rev. B, 74, 161403 (2006).

[22] E.V. Castro, K. Novoselov, S. Morozov, N. Peres, J.L. Dos Santos, J. Nilsson, F. Guinea, A. Geim, A.C. Neto. Phys. Rev. Lett., 99, 216802 (2007).

[23] A.C. Neto, F. Guinea, N.M. Peres, K.S. Novoselov, A.K. Geim. Rev. Mod. Phys., 81, 109 (2009).

[24] E. McCann, M. Koshino. Rep. Prog. Phys., 76, 056503 (2013).

[25] A.B. Kuzmenko, E. van Heumen, D. van der Marel, P. Lerch, P. Blake, K.S. Novoselov, A.K. Geim. Phys. Rev. B, 79, 115441 (2009).

[26] K. Majumdar, K.V.R.M. Murali, N. Bhat, Y.-M. Lin. Appl. Phys. Lett., 96, 123504 (2010).

[27] R. McGouran, M.M. Dignam. arXiv.org, cond-mat, arXiv:1701.00028 (2016).

[28] F.C. Moon. Chaotic Vibrations (N.Y., Wiley-Interscience Publication, Wiley \& Sons, 1987).

[29] Л.А. Фальковский. УФН, 178, 923 (2008).

\section{On the possibility of the solitary electromagnetic wave propagation in bigraphene}

\author{
E.I. Kukhar ${ }^{1,2}$, S.V. Kryuchkov ${ }^{1,2}$, E.S. Ionkina ${ }^{2}$ \\ ${ }^{1}$ Volgograd State Socio-Pedagogical University, \\ 400066 Volgograd, Russia \\ ${ }^{2}$ Volgograd State Technical University, \\ 400005 Volgograd, Russia
}

Abstract The possibility of the propagation of a solitary electromagnetic wave in the biased bilayer graphene had been studied. A condition of generation of such wave had been found. The dependence of the solitary wave shape on the potential difference between the graphene layers had been investigated. To the end of possible identification of the solitary wave the charge dragged by the wave along the direction of its propagation had been calculated.

Редактор Г.А. Оганесян 